\title{
On Wright's Inductive Definition of Coherence Truth for Arithmetic
}

\author{
JEFFREY KETLAND
}

\section{Wright's Notion of Coherence Truth}

As the first illustration of a potential satisfier for the 'platitudes for truth' in the appendix to his engaging recent discussion of the concept of truth (Wright 1999), Crispin Wright has proposed a notion of 'truth conceived as coherence' for arithmetic. This paper attempts to clarify certain aspects of Wright's proposal.

Take the standard first-order language of arithmetic $L{ }^{1}$ Let $B$ be some axiom system for arithmetic, which Wright calls the 'coherence base'. With small notational modifications, Wright proposes the following inductive definition of the concept 'coheres with $B$ ' (for $L$-sentences):

$\left(\mathbf{C}_{\mathrm{At}}\right) \quad$ If $\varphi$ is atomic, then $\varphi$ coheres with $B$ iff $B \vdash \varphi$.

$\left(\mathbf{C}_{\neg}\right) \neg \varphi$ coheres with $B$ iff $\varphi$ does not cohere with $B$.

$\left(\mathbf{C}_{\wedge}\right) \quad \varphi \wedge \psi$ coheres with $B$ iff $\varphi$ and $\psi$ cohere with $B$.

$\left(\mathbf{C}_{\vee}\right) \quad \varphi \vee \psi$ coheres with $B$ iff either $\varphi$ or $\psi$ coheres with $B$.

$\left(\mathbf{C}_{\rightarrow}\right) \quad \varphi \rightarrow \psi$ coheres with $B$ iff either $\varphi$ does not or $\psi$ does cohere with $B$.

$\left(\mathbf{C}_{\forall}\right) \quad \forall x \varphi$ coheres with $B$ iff, for each number $n, \varphi(\mathbf{n})$ coheres with $B$.

(C) $\exists) \quad \exists x \varphi$ coheres with $B$ iff, for some number $n, \varphi(\mathbf{n})$ coheres with $B$.

First, note that except for the basis clause $\left(\mathbf{C}_{\mathrm{At}}\right)$ this is the same as the usual Tarskian inductive definition of truth for arithmetic: ${ }^{2}$

$\left(\mathbf{T}_{\mathrm{At}}\right) \quad$ If $\varphi$ has the form $t=u$, then $\varphi$ is true iff $\operatorname{val}(t)=\operatorname{val}(u){ }^{3}$

\footnotetext{
${ }^{1}$ The terms of $L$ are defined recursively from a basis of variables, the constant $\mathbf{0}$ and the operation symbols $s,+$ and $\times$. The numerals of $L$ are written $\mathbf{n}$, meaning $\mathbf{0}$ prefixed by $n$ occurrences of the successor symbol $s$. The atomic formulas of $L$ are equations of the form $t=u$ (with $t, u$ terms) and complex formulas of $L$ are defined by recursion on complexity as usual. Below, $\operatorname{Sent}(L)$ is the set of $L$ sentences of $L, \operatorname{AtSent}(L)$ is the set of atomic $L$-sentences and Form $(L)$ is the set of $L$-formulas. In the arithmetic formalization of semantics, we use $\operatorname{Sent}_{L}(x)$ and $\operatorname{ClTm}_{L}(x)$ to mean arithmetic formulas expressing respectively that $x$ is (the code of) a sentence of $L$ or that $x$ is (the code of) a closed term of $L$.

${ }^{2}$ See, for example McGee 1991, p. 72 or Feferman 1991, §2.3.2.
} 
$\left(\mathbf{T}_{\neg}\right) \neg \varphi$ is true iff $\varphi$ is not true.

$\left(\mathbf{T}_{\wedge}\right) \varphi \wedge \psi$ is true iff $\varphi$ and $\psi$ are both true.

( $\left.\mathbf{T}_{\vee}\right) \quad \varphi \vee \psi$ is true iff either $\varphi$ or $\psi$ is true.

$\left(\mathbf{T}_{\rightarrow}\right) \varphi \rightarrow \psi$ is true iff either $\varphi$ is not true or $\psi$ is true.

$\left(\mathbf{T}_{\forall}\right) \quad \forall x \varphi$ is true iff, for each number $n, \varphi(\mathbf{n})$ is true.

( $\left.\mathbf{T}_{\exists}\right) \quad \exists x \varphi$ is true iff, for some number $n, \varphi(\mathbf{n})$ is true.

Any inductively defined set of $L$-sentences can be explicitly defined as the smallest set fulfilling the given conditions. For the Tarskian inductive definition $\left(\mathbf{T}_{\mathrm{At}}\right)-\left(\mathbf{T}_{\exists}\right)$, the smallest such set is the set of arithmetic truths $\operatorname{Th}(\mathbf{N})$ : the set of $L$-sentences true in the standard structure $\mathbf{N}$.

In light of Wright's definition, consider the following inductive definition.

Definition A: Let $B$ be an r.e. theory in $L$. Then let a set $X \subseteq \operatorname{Sent}(L)$ be called a Wright set for $B$ just in case, it satisfies the following conditions:

(i) $\varphi \in \operatorname{AtSent}(L) \Rightarrow(\varphi \in X \Leftrightarrow B \vdash \varphi)$

(ii) $\neg \varphi \in X \Leftrightarrow \varphi \notin X$

(iii) $\varphi \wedge \psi \in X \Leftrightarrow(\varphi \in X$ and $\psi \in X)$

(iv) $\varphi \vee \psi \in X \Leftrightarrow(\varphi \in X$ or $\psi \in X)$

(v) $\varphi \rightarrow \psi \in X \Leftrightarrow(\varphi \notin X$ or $\psi \in X)$

(vi) $\forall x \varphi \in X \Leftrightarrow$ for each number $n, \varphi(\mathbf{n}) \in X$

(vii) $\exists x \varphi \in X \Leftrightarrow$ for some number $n, \varphi(\mathbf{n}) \in X$.

If there exists a unique set $X$ satisfying these conditions, it is denoted $W_{B}$.

Given a coherence base $B$, the sets that Wright inductively defined have the form $W_{B}$. The uniqueness of any such set $W_{B}$ (if it exists) is established by an induction on complexity of formulas. We see in a moment that there are choices of $B$ for which $W_{B}$ doesn't exist. In general, the set $W_{B}$ depends by clause (i) only on the atomic sentences provable in $B$. So, if $B$ and $B^{*}$ prove the same atomic sentences, then $W_{B}=$

\footnotetext{
${ }^{3}$ Here val(.) is the recursive valuation function such that for any closed term $t, \operatorname{val}(t)$ is the number $n$ that the term $t$ designates. For further details see Kaye 1991 or Hájek and Pudlák 1991.
} 
$W_{B^{*}}$. By the negation clause (ii), $W_{B}$ must be complete and consistent. The clauses (ii)-(vii) together guarantee that $W_{B}$ is closed under the first-order consequence operation Cn. So $W_{B}$ is also a complete, consistent theory in $L$.

We first prove a surprising result ${ }^{4}$ concerning the existence of such sets $W_{B}$. It is necessary first to define what it means for a theory $B$ in $L$ to have "numerical witnesses". We say that the numeral $\mathbf{n}$ is a witness in $B$ for a closed term $t$ just in case $B \vdash t=\mathbf{n}$. If every term $t$ has a witness in $B$, we say that $B$ has numerical witnesses. Otherwise, $B$ lacks a numerical witness. If $B$ has numerical witnesses, then for all $n$, $\psi(\mathbf{n}) \in W_{B}$ if and only if for all closed terms $t, \psi(t) \in W_{B}$.

Theorem 1: If $B$ lacks a numerical witness, no set $X$ satisfies Definition A.

Proof: For reductio, assume such a set $X$ exists. By (ii), it must be consistent. But there is a closed term $t$ such that $B$ does not prove $t=\mathbf{n}$ (for any $n$ ). Hence, by (i), $t=$ $\mathbf{n} \notin X$, for all $n$. So, for all $n, t \neq \mathbf{n} \in X$. So, by (vi), $\forall x(t \neq x) \in X$. Since $X$ is closed under the consequence relation, we have $t \neq t \in X$, contradicting the consistency of $X$. Hence, there is no such set $X$. QED.

Before we prove the following results, we introduce the idea from model theory of a canonical model for $=$-closed set $Y$ of atomic sentences of $L$ (for details, see Hodges 1997, pp. 19-21). Let $Y$ be an $=$-closed set of atomic sentences of $L$. That is, each formula $t=t \in Y$; and if $t=u \in Y$ then $u=t \in Y$; and if $t=u \in Y$ and $u=v \in \mathrm{Y}$, then $t=v \in Y$. We define the canonical model $\mathbf{M}$ for $Y$ as follows. For any closed terms $t$, $u$ define an equivalence $\approx_{Y}$ so that $t \approx_{Y} u$ just in case $t=u \in Y$. The relation $\approx_{Y}$ is an equivalence relation because $Y$ is $=$-closed. Define $[t]$ as $\left\{u: u \approx_{Y} t\right\}$. Define an $L$ structure $\mathbf{M}$ with $\operatorname{dom}(\mathbf{M})=\{[t]: t$ is a term in $L\}$ and operations $S, \oplus, \otimes$ on $\operatorname{dom}(\mathbf{M})$ given by,

(a) $[t]=S([u])$ just in case $t=s(u) \in Y$

(b) $[t]=\left[u_{1}\right] \oplus\left[u_{2}\right]$ just in case $t=u_{1}+u_{2} \in Y$

(c) $[t]=\left[u_{1}\right] \otimes\left[u_{2}\right]$ just in case $t=u_{1} \times u_{2} \in Y$

In this $L$-structure $\mathbf{M}$, we interpret the constant $\mathbf{0}$ as [0], and the function symbols s, + and $\times$ as these operations $S, \oplus$ and $\otimes$. By induction on the complexity of terms, we

\footnotetext{
${ }^{4}$ This result partly due to Robert Black.
} 
can show that, for each term $t, t^{\mathbf{M}}=[t]$. So, each element of $\operatorname{dom}(\mathbf{M})$ has the form $t^{\mathbf{M}}$. For any atomic sentence $\varphi$, we have $\mathbf{M} \vDash \varphi$ if and only if $\varphi \in Y$. Finally, it follows that $\mathbf{M} \vDash \forall x \varphi(x)$ if and only if, for each term $t, \mathbf{M} \vDash \varphi(t)$.

Using this construction, the following theorem characterizes the sets inductively defined by Definition A above:

Theorem 2: Let $B$ have numerical witnesses, let $Y$ be the set of atomic sentences provable in $B$ and let $\mathbf{M}$ be the canonical model for $Y$. Then $W_{B}=\operatorname{Th}(\mathbf{M})$.

Proof: Let $X$ abbreviate $W_{B}$, let $Y$ be the set of atomic sentences provable in $B$. Clearly, $Y$ is =-closed. Let $\mathbf{M}$ be the canonical model for $Y$. Then $\varphi \in Y$ if and only if $\mathbf{M} \vDash \varphi$. The fact that each term has a numerical witness implies that, for each $[t] \in$ $\operatorname{dom}(\mathbf{M}),[t]=[\mathbf{n}]$, for some $n$. Hence, $\mathbf{M} \vDash \forall x \varphi(x)$ if and only if, for all $n, \mathbf{M} \vDash \varphi(\mathbf{n})$. We now show by induction that $W_{B}=\operatorname{Th}(\mathbf{M})$. That is, we show that, for each $\varphi \in$ Sent $(L)$, we have $\varphi \in X$ if and only if $\mathbf{M} \vDash \varphi$. Suppose $\varphi$ is $t=u$. Then $\varphi \in X$ if and only if $B \vdash \varphi$, iff $\varphi \in Y$, iff $\mathbf{M} \vDash \varphi$, as required. Let $\varphi$ have the form $\neg \psi$ and suppose that $\psi \in X$ if and only if $\mathbf{M} \vDash \psi$. Then $\varphi \in X$ if and only if $\neg \psi \in X$, iff $\psi \notin X$, iff not:-(M $\vDash \psi)$, iff $\mathbf{M} \vDash \neg \psi$, iff $\mathbf{M} \vDash \varphi$, as required. The cases for the other logical connectives are similar. Finally, let $\varphi$ have the form $\forall x \psi(x)$ and suppose that for all $t$, $\psi(t) \in X$ if and only if $\mathbf{M} \vDash \psi(t)$. Then, $\varphi \in X$ if and only if, for all $n, \psi(\mathbf{n}) \in X$, iff for all $t, \psi(t) \in X$, iff for all $t, \mathbf{M} \vDash \psi(t)$, iff $\mathbf{M} \vDash \forall x \psi(x)$, iff $\mathbf{M} \vDash \varphi$, as required. The subcase where $\varphi$ is existentially quantified is similar. QED.

An immediate corollary of Theorem 2 is:

Corollary 3: If $B$ is inconsistent, then $W_{B}$ is the theory of the single element model, axiomatized by the sentence $\forall x(x=\mathbf{0})$.

Proof: $B$ proves every atomic sentence, so $B$ has numerical witnesses for each term and Theorem 2 applies. Let $Y$ be the set of atomic sentences provable in $B$. Clearly, for each term $t, t=\mathbf{0} \in Y$. So, $t \approx \mathbf{0}$, and thus $[t]=[\mathbf{0}]$. The domain of the canonical model thus has one element, [0]. Furthermore, the operations are defined by the conditions: $S([\mathbf{0}])=[\mathbf{0}]$ and $[\mathbf{0}] \oplus[\mathbf{0}]=[\mathbf{0}]$ and $[\mathbf{0}] \otimes[\mathbf{0}]=[\mathbf{0}]$. The theory of this model is the complete consistent theory axiomatized by the sentence $\forall x(x=\mathbf{0})$. QED. 
It is well-known that the standard structure $\mathbf{N}$ for arithmetic is isomorphic to the canonical model for the set of true atomic sentences of arithmetic. Hence,

Theorem 4: Let $B$ be sound and complete for atomic sentences. Then $W_{B}=\operatorname{Th}(\mathbf{N})$.

This means that Wright's inductively defined notion of coherence truth is coextensive with the standard Tarskian definition provided the coherence base $B$ proves all the true atomic sentences and no false ones. This proviso is clearly a strong constraint, since for any particular choice of $B$, the meta-proof that 'coherence truth' with respect to $B$ is co-extensional with arithmetic truth will require a proof of $B$ 's consistency. See below for an extended discussion of this.

Fortunately, even the very weakest formal systems of arithmetic of any interest are sound and complete for atomic sentences. Indeed, the set of atomic truths of arithmetic is decidable. For example, an electronic calculator computes the value of any closed term $t$, subject to memory resources and the boredom or stamina of the human inquirer. The property of decidability is lost at the next step up in the KleeneMostowski hierarchy, although one may still have $\Sigma_{1}$-completeness, where a $\Sigma_{1}$ sentence has the form $\exists x_{1} \ldots x_{n} R\left(x_{1}, \ldots, x_{n}\right)$, with $R$ a recursive predicate. That is, there are many sound formal systems which prove all $\Sigma_{1}$-truths. For example, Robinson arithmetic $Q$ is sound and $\Sigma_{1}$-complete. The property of completeness is lost at the level of $\Pi_{1}$-truths: Gödel's first incompleteness theorem implies that no sound r.e. system of arithmetic extending $Q$ is $\Pi_{1}$-complete.

In any case, because $Q$ proves all atomic truths and refutes all atomic falsehoods,

Theorem 5: Let $B$ be any consistent extension of $Q$. Then $W_{B}=\operatorname{Th}(\mathbf{N})$.

So, the set of $L$-sentences 'cohering' with any such base theory $B$ is precisely the set of arithmetic truths.

\section{The Material Adequacy of Wright's Proposal}

Wright asks whether the Tarski T-sentences are derivable from his inductive definition:

... does this proposal validate the equivalence scheme? Can it be affirmed, for all first-order expressible arithmetical statements $p$, that

(EC) $p$ coheres with $B$ if and only if $p$ ? 
(Wright 1999, p. 235).

The answer is Yes, but with some important qualifications. Any truth theory $\Gamma$ in the language $L_{T}$ (the extension of $L$ formed by adding a primitive monadic predicate symbol T) containing a sufficient amount of formalized syntax, plus the Tarskian inductive definition of truth, proves each base language instance of the disquotational $\mathrm{T}$-scheme. In fact, each instance of the uniform disquotation T-scheme is provable. Consider the standard Tarskian truth theory $\operatorname{Tr}(P A)$ consisting of $P A$ plus the natural formalization of the Tarski inductive axioms $\left(\mathbf{T}_{\mathrm{At}}\right)-\left(\mathbf{T}_{\exists}\right) .{ }^{5}$ Then, for each formula $\varphi(\underline{x})$ $\in \operatorname{Form}(L)$, we have ${ }^{6}$,

$$
\operatorname{Tr}(P A) \vdash \forall \underline{x}\left[T\left(\varphi(\underline{x})^{\rceil}\right) \leftrightarrow \varphi(\underline{x})\right] .
$$

This is established by a meta-induction on formula complexity. Then, in the case where the $L$-formula $\varphi$ has no free variables, this gives the usual Tarski T-sentences, of the form $T\left(\varphi^{\top}\right) \leftrightarrow \varphi$.

We can also formalize Wright's inductive definition. Since the coherence base $B$ is recursively axiomatized, the recursive relation " $n$ is the code of a proof in $B$ of formula with code $m$ " may be represented arithmetically by a $\Delta_{1}$-formula $\operatorname{Proof}_{B}(y, x)$. Let the standard provability predicate $\operatorname{Bew}_{B}(x)$ for $B$ be $\exists y \operatorname{Proof}_{B}(y, x)$. We extend the language $L$ of arithmetic by adding a primitive predicate $\operatorname{Coh}_{B}(x)$, to express Wright's coherence notion of truth. Then we can formalize Wright's inductive definition in the extended language $L+\left\{\operatorname{Coh}_{B}\right\}$ by the following axioms ${ }^{7}$,

$$
\begin{aligned}
& \left(\mathbf{C}_{\mathrm{At}}\right) \quad \operatorname{AtSent}_{L}(x) \rightarrow\left(\operatorname{Coh}_{B}(x) \leftrightarrow \operatorname{Bew}_{B}(x)\right) . \\
& \left(\mathbf{C}_{\neg}\right) \quad \operatorname{Coh}_{B}(\neg(x)) \leftrightarrow \neg \operatorname{Coh}_{B}(x) . \\
& \left(\mathbf{C}_{\wedge}\right) \quad \operatorname{Coh}_{B}(x \wedge y) \leftrightarrow\left(\operatorname{Coh}_{B}(x) \wedge \operatorname{Coh}_{B}(y)\right) . \\
& \left(\mathbf{C}_{\vee}\right) \quad \operatorname{Coh}_{B}(x \vee y) \leftrightarrow\left(\operatorname{Coh}_{B}(x) \vee \operatorname{Coh}_{B}(y)\right) .
\end{aligned}
$$

\footnotetext{
${ }^{5}$ This is the Tarskian theory of truth discussed, for example, in Feferman 1991 and Halbach 1996. This theory is sometimes called $P A(S)$. See Halbach 2000 'Truth and Reduction' for a comprehensive survey of recent work on axiomatic truth theories.

${ }^{6}$ The sentence $\forall \underline{x}\left[T\left(\varphi(\underline{x})^{\rceil}\right) \leftrightarrow \varphi(\underline{x})\right]$ is the uniform Tarski disquotation sentence for $\varphi(\underline{x})$, where $\underline{x}$ is a finite sequence of variables and (boldface) $x$ is a defined function term with the variable $x$ free meaning 'the code of the result of substituting the standard numeral for $x$ '.

${ }^{7}$ The notations ' $\neg(x)$ ', ' $x \wedge y$ ', $\ldots, ' \forall(x, v)$ and ' $x(v / y)$ ' below are function symbols, which are arithmetizations of the syntactic notions needed to formalize Wright's clauses. For example, ' $\neg(x)$ ' means 'the code of the formula whose negation has code $x$ ' and ' $\forall(x, v)$ ' means 'the code of the universal quantification of the formula whose code is $x$ with respect to the variable whose code is $v$ '.
} 
$\left(\mathbf{C}_{\rightarrow}\right) \quad \operatorname{Coh}_{B}(x \rightarrow y) \leftrightarrow\left(\operatorname{Coh}_{B}(x) \rightarrow \operatorname{Coh}_{B}(y)\right)$

$\left(\mathbf{C}_{\forall}\right) \quad \operatorname{Coh}_{B}(\forall(x, v)) \leftrightarrow \forall y \operatorname{Coh}_{B}(x(v / y))$

$\left(\mathbf{C}_{\exists}\right) \quad \operatorname{Coh}_{B}(\exists(x, v)) \leftrightarrow \exists y \operatorname{Coh}_{B}(x(v / y))$.

(ß) $\operatorname{Coh}_{B}(x) \rightarrow \operatorname{Sent}_{L}(x)$

We have seen that the co-extensiveness of the set $W_{B}$ and the set of arithmetic truths amounts to the assumption that the atomic sentences provable in $B$ are precisely those which are true. Since this entails that $B$ does not prove $\mathbf{0}=\mathbf{1}$, this assumption implies the consistency of $B$. So, our coherence base $B$ had better not be too strong. We should not want our meta-theoretic verification that theory of truth is materially adequate to bear the further quite unwarranted burden of proving the consistency of $B$ if $B$ is strong.

Definition B: $P A\left(\mathrm{Coh}_{B}\right)$ is the theory whose axioms are the axioms of $P A$ plus the coherence axioms $\left(\mathbf{C}_{\mathrm{At}}\right)-(\beta)$.

By a modification of what we already know about the Tarskian truth theory $\operatorname{Tr}(P A)$, we have the following result:

Theorem 6. $P A\left(C o h_{B}\right)$ is materially adequate just in case it proves the atomic reflection principle,

$$
\left(\operatorname{AtRfn}_{B}\right) \quad \forall t, u \in \operatorname{ClTm}_{L}\left[\operatorname{Bew}_{B}(t=u) \leftrightarrow \operatorname{val}(t)=\operatorname{val}(u)\right] .
$$

Reflection principles are generically stronger than the theory upon which they reflect. Assuming $P A$ as background theory, $\left(\operatorname{AtRfn}_{B}\right)$ implies $\operatorname{Bew}_{B}\left(\mathbf{0}=\mathbf{1}^{\dagger}\right) \leftrightarrow$ $\operatorname{val}\left({ }^{[} \mathbf{0}^{\top}\right)=\operatorname{val}\left(\mathbf{1}^{\top}\right)$, which implies $\operatorname{Bew}_{B}\left(\mathbf{0}^{\top}=\mathbf{1}^{\top}\right) \leftrightarrow \mathbf{0}=\mathbf{1}$, and thus $\neg \operatorname{Bew}_{B}\left(\mathbf{0}=\mathbf{1}^{\top}\right)$. Hence,

Corollary 7: If $P A\left(\mathrm{Coh}_{B}\right)$ is materially adequate, then it implies $\operatorname{Con}(B)$.

Thus, a coherence truth theory constructed along Wright's lines is materially adequate only if it implies the atomic reflection principle (and thus the consistency) of the coherence base $B$. It would therefore be problematic to take $B$ as the (r.e.) set of arithmetic sentences (under some standard interpretation of arithmetic in set theory) provable in $Z F C$, since we are none too sure of the consistency of $Z F C$. Indeed, it would be entirely redundant, as noted before, since rather weak arithmetic theories such as $Q$ already imply all the atomic truths. Actually, the situation is even worse. 
For $P A$ (if it is consistent, etc.) certainly does not imply the (arithmetic) atomic reflection principle for $Z F C$. Hence,

Corollary 8: Suppose $P A$ and $Z F C$ are consistent. Then $P A\left(C o h_{Z F C}\right)$ is not materially adequate.

The material adequacy of a theory of 'coherence' truth along these lines thus depends upon two conditions:

(i) The coherence base $B$ must be sound and complete for atomic sentences;

(ii) The atomic reflection principle $\left(\operatorname{AtRfn}_{B}\right)$ for the coherence base $B$ must be provable in the truth theory.

To satisfy these constraints, for example, the background theory can be chosen to be $P A$, and the coherence base chosen as some subsystem of $P A$ whose soundness and completeness for atomic sentences can be proved in $P A$. If we select the base to be $Q$, these requirements are met, since the atomic reflection principle for $Q$, stating that any atomic sentence is provable in $Q$ if and only if true, is provable in $P A$,

$$
\left(\operatorname{AtRfn}_{Q}\right) \quad \forall t, u \in \operatorname{ClTm}_{L}\left[\operatorname{Bew}_{Q}(t=u) \leftrightarrow \operatorname{val}(t)=\operatorname{val}(u)\right]
$$

This verifies the following corollary of Theorem 6:

Corollary 9: $P A\left(\mathrm{Coh}_{Q}\right)$ is materially adequate.

Theorem 6 indicates that we must be able to prove the standard Tarskian truth condition for atomic sentences (in our truth theory) in order to derive the T-sentences. This then suggests that Wright's approach to 'coherence' truth really is just getting the epistemic notion of provability and the semantic concept of truth back-to-front. For truth is a semantical concept, not an epistemic or proof-theoretic one. The reason why an atomic sentence of the form $t=u$ is true is intrinsically semantical: the semantic value of $t$ is identical to the semantic value of $u$. A sentence is not true because it is provable in some axiom system, unless of course this axiom system is already sound, and soundness is intrinsically semantical. There is no eliminating this irreducible semantical aspect.

Of course, the correct truth value of any atomic arithmetic sentence is always computable, and there exist weak axiom systems, such as $Q$, which prove all these truths and no falsehoods. It seems to me that this convergence of semantic truth value and epistemic computability of truth value for atomic sentences can be 
philosophically misleading, since the 'symmetry' of semantics and epistemology for basic arithmetic facts is 'broken' even at very low levels of logical complexity of assertions about the natural numbers. For example, at the level of $\Pi_{1}$-sentences. The reason is that the set of true $\Pi_{1}$-sentences is not recursively enumerable, and hence not recursively axiomatizable.

Wright continues:

Dialectically, the status of a positive answer is somewhat akin to that of Church's Thesis, that all effectively calculable arithmetic functions are general recursive. A formal proof of Church's Thesis would demand some independent formal characterization of the effectively calculable functions - the very thing that Church's Thesis purports to provide. Likewise a proof of (EC) would demand some independent characterization of the firstorder arithmetic truths. (Wright 1999, p. 235).

We have seen above how material adequacy, Wright's (EC), is proved. So I am unsure how to evaluate this claim. Theorem 5 establishes that the set of sentences 'cohering' with a base theory $B$ is precisely the same as the set of arithmetic truths if $B$ is in fact (although we might not be able to prove it) a consistent extension of $Q$. Theorem 6 establishes that if the base theory $B$ is not too weird and not too strong (say, a consistent extension of $Q$ whose consistency is provable in $P A$ ), then all the Tsentences are theorems of the system comprising $P A+$ the inductive definition of 'coheres with $B$ '.

However, the material adequacy of a Wright-style coherence truth theory, with base $B$, ultimately requires a proof that an atomic sentence $t=u$ is provable in $B$ if and only if $\operatorname{val}(t)=\operatorname{val}(u)$. The right-hand-side here is indeed an 'independent characterization of arithmetic truth' (for atomic sentences)—namely, Tarski’s.

Furthermore, Tarski's notion of a materially adequate truth definition/theory (i.e. one that proves all the disquotational T-sentences) does constrain the extension of the truth predicate rather tightly, modulo a certain subtlety concerning syntactic $\omega$ incompleteness. Consider a truth theory $\Gamma$ for the base language $L$ containing two materially adequate truth predicates, called $T$ and $T^{*}$, say. Then it is clear that we have, $\Gamma \vdash T\left(\varphi^{\top}\right) \leftrightarrow T^{*}\left(\varphi^{\top}\right)$, for each $\varphi \in \operatorname{Sent}(L)$. But $\omega$-incompleteness is pervasive, and material adequacy alone does not necessarily guarantee that the universal 
generalization expressing co-extensionality of $T$ and $T^{*}$ is provable. That is, material adequacy alone does not guarantee that $\Gamma \vdash \forall x\left[\operatorname{Sent}_{L}(x) \rightarrow\left(T(x) \leftrightarrow T^{*}(x)\right)\right]$.

The underlying reason is that there may be non-standard models for the syntactical component of the semantical theory $\Gamma$. In a non-standard model $M \vDash \Gamma$, the set $\operatorname{Sent}_{L}(M)$ of entities that the model $M$ 'believes' to be sentences of the base language $L$ may contain elements which are not codes of sentences of $L$. However, so long as the base model is standard, the expansion to a model of the truth axioms in unique.

This situation can be improved, as pointed out by Vann McGee (see McGee 1991, p. 73). Let $\Gamma$ be a formalized truth theory containing two 'truth' predicates $T$ and $T^{*}$ both governed by Tarski's inductive axioms. Suppose that $\Gamma$ expands the induction scheme to permit induction on formulas containing the truth predicates $T$ and $T^{*}$. Then we have $\Gamma \vdash \forall x\left[T(x) \leftrightarrow T^{*}(x)\right]$. We can use this to give a sort of uniqueness theorem. First,

Definition C: $P A\left(T, C_{B}\right)$ is the formalized truth theory combining the standard Tarskian inductive definition and Wright's inductive definition of coherence truth and the induction scheme is expanded to include all formulas of the extended language $L$ $+\left\{\operatorname{Coh}_{B}, T\right\}$.

We then obtain:

Theorem 10: Let $B$ be a coherence base such that $P A \vdash\left(\operatorname{AtRfn}_{B}\right)$. Then,

$$
P A\left(T, \operatorname{Coh}_{B}\right) \vdash \forall x\left[T(x) \leftrightarrow \operatorname{Coh}_{B}(x)\right]
$$

This suggests to me that a characterization of arithmetic truth independent of the Tarskian definition is not needed. Any definition of 'true sentence of arithmetic' must be materially adequate. Otherwise it is simply not a truth concept. And Tarski's inductive definition is materially adequate. This is part of the point of Tarski's Convention $\mathrm{T}$ and surely Tarski achieved a fundamental insight about how to place constraints on theories of truth:

... it is important to notice that the conditions for the material adequacy of the definition determine uniquely the extension of the term "true". Therefore, every definition of truth which is materially adequate would necessarily be equivalent to that actually constructed. The semantic conception of truth gives 
us, so to speak, no possibility of choice between various non-equivalent definitions of this notion. (Tarski 1944, 353-4).

Kings College, London

Strand, London WC2R 2LS

jeffrey.ketland@kcl.ac.uk

\section{References}

Blackburn, S. and K. Simmons. (eds.) 1999. Truth. Oxford: Oxford University Press.

Feferman, S. 1991. Reflecting on Incompleteness. Journal of Symbolic Logic 56: 149.

Hájek, P. and P. Pudlák 1991. Metamathematics of First-Order Arithmetic. Berlin: Springer.

Halbach, V. 1996. Axiomatische Wahrheitstheorien. Berlin: Akademie Verlag.

Halbach, V. 2000. Truth and Reduction. Erkenntnis 53: 97-126.

Hodges, W.1997. A Shorter Model Theory. Cambridge: Cambridge University Press.

Kaye, R. 1991. Models of Peano Arithmetic. Oxford Logic Guides 15. Oxford: Oxford University Press.

McGee, V. 1991. Truth, Vagueness and Paradox. Indianapolis: Hackett.

Tarski, A. 1935/6. Der Wahrheitsbegriff in den formalisierten Sprachen. Studia Philosophica 1: 261-405. English translation by J.H. Woodger, "The Concept of Truth in Formalized Languages", in A. Tarski 1956 Logic, Semantics and Mathematics. Oxford: Clarendon Press.

Tarski, A. 1944. The Semantic Conception of Truth and the Foundations of Semantics. Philosophy and Phenomenological Research 4: 341-76. Reprinted in Blackburn \& Simmons (eds.) 1999.

Wright, C. 1999. Truth: A Traditional Debate Reviewed. In Pragmatism, ed. C. Misak, Canadian Journal of Philosophy, Suppl. Vol. 24. Page references are to the reprint in Blackburn \& Simmons (eds.) 1999. 